Document downloaded from:

http://hdl.handle.net/10251/161858

This paper must be cited as:

Bocanegra, RA.; Vallés-Morán, FJ.; Francés, F. (2020). Review and analysis of vehicle stability models during floods and proposal for future improvements. Journal of Flood Risk Management. 13:1-13. https://doi.org/10.1111/jfr3.12551

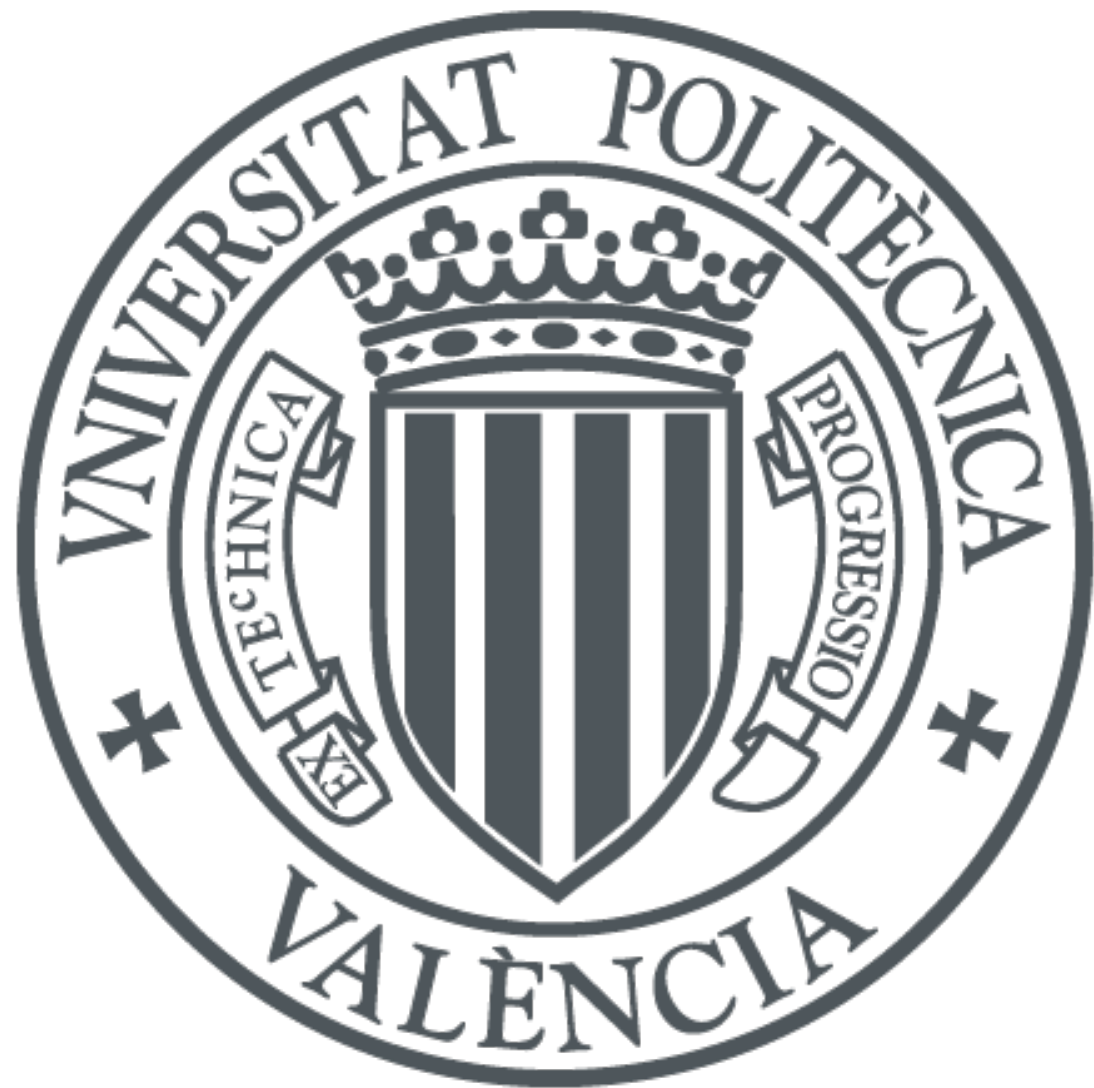

The final publication is available at

https://doi.org/10.1111/jfr3.12551

Copyright Wiley

Additional Information

This is the peer reviewed version of the following article: Bocanegra, RA, Vallés-Morán, FJ, Francés, F. Review and analysis of vehicle stability models during floods and proposal for future improvements. J Flood Risk Management. 2020; 13 ( Suppl. 1):e12551, which has been published in final form at https://doi.org/10.1111/jfr3.12551. This article may be used for non-commercial purposes in accordance with Wiley Terms and Conditions for SelfArchiving. 


\section{REVIEW AND ANALYSIS OF VEHICLE STABILITY MODELS DURING FLOODS AND PROPOSAL FOR FUTURE IMPROVEMENTS}

\section{Ricardo A. Bocanegra ${ }^{1}$, Francisco J. Vallés ${ }^{2}$ and Félix R. Francés ${ }^{3}$}

1 Universitat Politècnica de València - Instituto de Investigación en Ingeniería del Agua y Medio Ambiente (IIAMA), Spain, ribovi@doctor.upv.es. Corresponding author

2 Universitat Politècnica de València - Instituto de Investigación en Ingeniería del Agua y Medio Ambiente (IIAMA), Spain, fvalmo@hma.upv.es

3 Universitat Politècnica de València - Instituto de Investigación en Ingeniería del Agua y Medio Ambiente (IIAMA), Spain, ffrances@hma.upv.es 


\title{
REVIEW AND ANALYSIS OF VEHICLE STABILITY MODELS DURING FLOODS AND PROPOSAL FOR FUTURE IMPROVEMENTS
}

\begin{abstract}
Flood water can affect vehicles significantly, which in turn can increase the negative effects of floods as vehicles are washed away by the flow and become a form of debris. In cities, most fatalities during floods occur inside vehicles. Consequently, it is necessary to establish thresholds for vehicle stability during this type of event to provide information necessary for flood risk management. This article analyses the available stability models developed over recent years to determine such thresholds. The stability models were grouped according to the way in which they approached car watertightness and the stability thresholds proposed by each of them were compared. It was found that these thresholds vary over a relatively wide range. Additionally, the experimental data were compared with the results provided by these studies leading to the conclusion that several of the stability models analysed do not fit measured data well. New research is required to overcome the simplifications made by the state-of-the-art models and to try to standardize the decision criteria which should be adopted to define stability thresholds for vehicles of different characteristics.
\end{abstract}

KEY WORDS: Vehicle stability; Floods; Car watertightness; Safety criteria

\section{INTRODUCTION}

River overflows can significantly affect transport systems due to the blockage of roads and the risk they generate for vehicles being driven or parked on floodplains (Teo et al. 2012a; Versini et al. 2010a). Vehicles can be washed away by overflowing water bodies, effectively becoming debris that can cause additional damage by impacting buildings and infrastructure and by clogging hydraulic structures (Teo et al. 2012b; Kalantari et al. 2014; Arrighi et al. 2015; Pregnolato et al. 2017). Vehicles are swept along during floods much more frequently than it seems, even in large numbers in some cases. In August of 2004, a flash flood affected the village of Boscastle in the United Kingdom, causing enormous damage and sweeping away more than 100 vehicles, some of which blocked a bridge causing its collapse while driving others to the sea (Teo et al. 2012a; Teo et al. 2012b). In August 2009, a flash flood occurred in the city of Chongquin in China, taking five cars parked on the floodplain to the Yangtze River (Xia 2014). In October 2014 the city of Santa Cruz de Tenerife, Spain, a large number of vehicles were flooded and swept away by a big flood (Martínez-Gomariz et al. 2017).

The main cause of death in cities during flood events corresponds to cars being swept away when they are driven by flooded roads (Jonkman and Kelman 2005; Drobot et al. 2007; Fitzgerald et al. 2010; Kellar and Schmidlin 2012). In developed countries a high percentage of these deaths occurs during flash floods when drivers try to cross overflowing water bodies instead of avoiding them (Fitzgerald et al. 2010; Kellar and Schmidlin 2012). Hence, in areas subject to flash floods almost half of the victims are passengers trapped inside their own vehicles (Versini et al. 2010a).

In addition to direct damages, which include fatalities, vehicle damages and the exacerbation of the negative consequences floods have on infrastructure, swept away vehicles also generate indirect damage due to traffic disruption. Additionally, the rescue of people from flooded cars demands a significant amount of time and resources (Smith et al. 2017). 
However, despite the danger caused by vehicles swept away and the fact that once vehicles have been washed away they can aggravate flood impacts, very few studies have been carried out on this topic so far (Suárez et al. 2005; Teo et al. 2012a; Arrighi et al. 2015). Most of the available studies were conducted in laboratory flumes during the 60's and 70's while some theoretical analyses were done in the 90's. However, given the significant changes undergone by vehicles over the last decades, it is considered that these studies are not representative of current conditions anymore (Arrighi et al. 2015; Teo et al. 2012a). A review of the state of the art was presented by Martínez - Gomariz et al. (2016a). The present article develops an indepth analysis of some of the methodologies presented in that study and includes the methodologies developed over the last years.

This article discusses the existing models developed in recent years to establish stability thresholds that can be applied to one or more types of vehicles according to their authors. In order to do this, a description of these stability models is made first, grouping them according to how they consider vehicle watertightness. Subsequently, the stability thresholds proposed by these models are inter-compared, based on the type of cars for which they were developed. Later, the ranges in which these stability thresholds fluctuate are compared with the pairs of velocity and depth data measured in laboratory for which the studied cars became unstable. Finally, the conclusions highlight some of the topics on which further research should concentrate to develop models that allow vehicle stability thresholds to be defined with greater precision.

\section{DESCRIPTION OF THE STUDIED STABILITY MODELS}

Loss of stability in a vehicle can be generated by the hydrodynamic mechanisms of floating and sliding (Arrighi et al. 2015). Loss of floating stability occurs when buoyancy and lift effect exceed vehicle weight, causing for most cases the rear wheels to lose traction, due to weight distribution in modern vehicles, making the vehicle rotate on its front wheels and in many cases be washed away by the flow. This type of instability occurs mainly when the flow moves at a slow velocity and high depths of water are found. Loss of sliding stability occurs when the drag force exerted by the flow exceeds frictional force. Floating and sliding mechanisms interact with each other, since the buoyancy and lift effect reduce the normal component of the weight, reducing the force that opposes the drag one.

Another potential failure mechanism obeys to toppling, which seems to occur only when the vehicles have already been washed away by the flow or have floated and found irregular land (Shand et al. 2011). None of the available stability models to date has considered this type of instability.

In recent years, some research has been conducted with the objective of establishing a stability threshold for modern vehicles through the study of the interaction between these vehicles and the flow. This threshold is usually defined through expressions that relate water depth and flow velocity. However, many of these studies differ in the way they approach the problem and in the driving factors considered in their analysis. As a result, they have produced different models for the determination of this stability threshold.

According to some authors (Teo et al. 2012a,b), assuming that vehicles are completely watertight during floods is a highly idealized condition, which is why they consider the entry of water into vehicles when trying to determine the stability threshold. However, most authors 
consider that, due to improvements in modern vehicles in aspects such as sealing against dust, it is legitimate to assume that vehicles are watertight during flood events. Some of them determine the stability threshold using the total energy of the flow (Ausroads 2008; Kramer et al. 2016) while others use the product of flow velocity and depth. Among the latter, some models establish a maximum limit for depth and flow velocity (DIPNR 2005; Shand et al. 2011; Smith et al. 2014) and others a maximum limit only for depth (Moore and Power 2002; Martínez-Gomariz et al. 2017). Finally, some authors have developed models that enable the calculation of vehicle stability either considering or disregarding its watertightness; i.e., one of these models establishes the stability threshold by registering the combinations of flow velocity and depth that generate stability loss (Toda et al. 2013), another compares the forces acting on the vehicle (Oshikawa and Komatsu 2014) and the third uses the Froude number and a mobility parameter (Arrighi et al 2015).

The different models available for evaluating stability of vehicles exposed to flooding are described in more detail here below. A synthesis of the main characteristics of these stability models is presented in Table 1 .

\subsection{Stability models that consider non-watertightness of vehicles during floods}

In order to understand the impact of water level and flow velocity in the hydrodynamic processes that cause stability loss of a vehicle during a flood event, Teo et al. (2012a) and Teo et al. (2012b) presented in similar papers a series of experiments in a flume using physical models of three different vehicle types: a Mini Cooper, a BMW M5 and a Mitsubishi Pajero. They used scales 1:43 and 1:18, satisfying the principle of geometric similarity. Additionally, these authors assumed the vehicles were not completely watertight. These experimental data were also reported by Xia et al. (2011).

Teo et al. (2012a, b) extrapolated their results to the prototypes and established the stability threshold from the combinations of water depth and flow velocity that cause the movement of the vehicles. On the basis of the obtained results, a graph was developed by relating depth with flow velocity, this graph enabled the definition of three zones: a stable zone where the interaction of flow velocities and depths does not affect vehicle stability, a transition zone and an unstable zone in which flow velocities and depths would cause the vehicles to move by sliding (Figure 1a).

However, according to _Froude number similarity, the weights of the different scale physical models were not correctly scaled. Consequently, the validation carried out for the 1:18 scale was not sufficiently accurate. Additionally, they considered water came inside the vehicle from the very beginning of the experiment whereas it appears the entry of water into the prototypes is likely to occur not as fast as it was assumed. Owing to all this, the results presented by this model should be used with caution.

\subsection{Stability models that consider vehicle watertightness during floods}

From the analysis of the experimental results reported by Bonham and Hattersley (1967), Gordon and Stone (1973) and Keller and Mitsch (1993), Moore and Power (2002) defined the threshold of instability through a linear relationship between flow velocity and depth for subcritical regime and through the multiplication of these two parameters for supercritical regime, assigning to this product a value of 0.6 (Figure 1b). They established the separation between these relationships at $1.81 \mathrm{~m} / \mathrm{s}$. It should be noted that this stability model was based 
on experimental tests carried out with vehicles having very different characteristics from current ones, so the results may not be entirely valid today.

The Department of Infrastructure, Planning and Natural Resources of the New South Wales Government (DIPNR 2005) considers that vehicle instability is initially generated by buoyancy and establishes a stability threshold through a linear relationship between flow depth and velocity. This threshold includes maximum values of $2.0 \mathrm{~m} / \mathrm{s}$ for the velocity and $0.3 \mathrm{~m}$ for the depth (Figure $1 \mathrm{~b}$ ).

Mens et al. (2008) obtained the stability thresholds for a standard car, a van, an ambulance and a fire truck applying the stability model proposed by Keller and Mitsch (1993), as shown in Figure 1b. However, as already noted, the characteristics of vehicles have changed significantly in recent years, so the model applied could not be valid at present.

Based on the analysis of the data reported by Bonham and Hattersley (1967), Gordon and Stone (1973) and Keller and Mitsch (1993), Australian Rainfall and Runoff -AR\&R- (Shand et al. 2011) proposed provisional stability criteria for vehicles at rest (Figure 1b). According to their dimensions, weight and free distance to the ground, the cars were classified into large 4WD, large passenger and small passengers (Sections 3 and 4 use this same classification) and it was considered that the stability limit for each of these types of vehicle is reached when the product of flow velocity with depth is equal to $0.3,0.45$ and 0.6 , respectively. According to buoyancy limits, maximum depths of $0.5 \mathrm{~m}$ were defined for large $4 \mathrm{WD}$ vehicles, $0.4 \mathrm{~m}$ for large passenger vehicles and $0.3 \mathrm{~m}$ for small passenger vehicles. A maximum flow velocity of $3.0 \mathrm{~m} / \mathrm{s}$ was established for all vehicles to ensure human safety when leaving the vehicles, following the recommendation reported by Cox et al. (2010). According to the authors, these criteria have a provisional character and must, therefore, be updated.

From results reported in literature, Smith et al. (2014) proposed a stability threshold for small vehicles and another for all other types of vehicle, considering in both cases a maximum limit of flow velocity of $2.0 \mathrm{~m} / \mathrm{s}$ (Figure 1b). The other criteria for both small and other vehicles coincide with the ones defined by the AR\&R in 2011 (Shand et al. 2011) for small vehicles and large 4WD vehicles, respectively.

Kramer et al. (2016) conducted several laboratory tests using a 1: 9.8 scale physical model of a VW Golf III and a 1:13.1 scale physical model of an emergency rescue vehicle. The results indicated that the different combinations of flow velocity and depth that define the stability threshold of the analysed vehicles describe a curve similar in shape to the curve of constant total energy head. These authors established that the safety criteria for the transit of vehicles on flooded roads must consider technical restrictions of each vehicle, such as the height of the air inlets or the tightness of the electrical devices, in addition to stability aspects. Consequently, a stability threshold equal to the total energy of the water was defined, giving it a constant value equivalent to the minimum wading depth, according to the vehicle under study: for emergency rescue vehicles this value was established at $0.6 \mathrm{~m}$ and for passenger vehicles at $0.3 \mathrm{~m}$. This last criterion coincides with that proposed by Ausroads in 2008 (Figure 1b).

Concerning the instability drivers, Kramer et al. (2016) concluded that in floods in which the Froude number of the flow is less than 0.5 , stability is controlled by the flotation forces and does not seem to depend on the orientation of the vehicle with respect to the flow. In contrast, 
when Froude numbers are greater than 0.5 , the sliding instability mechanism becomes more dominant and the incidence angle of the flow has an important effect.

Finally, through tests carried out with a prototype of the VW Golf III car, Kramer et al. (2016) concluded that it is reasonable to assume watertightness conditions in order to define safety criteria for vehicles in urban environments.

Smith et al. (2017) conducted measurements on a 2006 Toyota Yaris Sedan and a 1998 Nissan Patrol GRII on a full prototype scale in order to determine the force required to overcome the friction force when the vehicles were submerged at different depths of water at rest. Additionally, they conducted tests on a 1:18 scale physical model of a 2005 Toyota Yaris Hatch with the objective of determining the equivalent hydrodynamic force required to reproduce the instability conditions of the prototype vehicle. The test results showed average values of 0.76 for the friction coefficient between the floor and the tyres and values fluctuating between 1.2 and 2.0 for the drag coefficient, which is used to calculate the drag force. Stability thresholds were defined as the product of flow velocity and depth, finding values close to 0.5 for the Toyota Yaris and higher than 1.0 for the Nissan Patrol. However, considering that conditions in real world can differ widely from the controlled conditions in the laboratory and that several simplifications were made in the tests performed, Smith et al. concluded that the stability thresholds proposed by AR\&R (Shand et al. 2011) are appropriate (Figure 1b).

Martínez-Gomariz et al. (2017) proposed a model to determine the stability of any vehicle exposed to flooding based on the analysis of the results of experimental tests. Measurements were made with 12 car physical models using three different scales (1:14, 1:18 and 1:24). From the results, these authors defined a stability function that allowed them to establish a constant value of the product of flow velocity and depth. This function was found on the basis of the depth from which the vehicle starts to float and a stability coefficient which is calculated from the friction coefficient between the tyres and the road and the following characteristics of the vehicle: weight, free distance to the ground and plan area (Figure 1b).

Through the implementation of the obtained stability function by Martínez-Gomariz et al. (2017) and using friction coefficient values of 0.25 and 0.75 , the model enables to obtain a graph showing depth versus flow velocity for each vehicle. This graph shows a stable zone, a transition zone and a zone in which vehicles would reach instability conditions. However, it should be noted that in all tests performed, the friction coefficient fluctuated between 0.52 and 0.62 , while for the calculation of the stability thresholds the values adopted were of 0.25 and 0.75 , which are very far from the experimental range.

\subsection{Stability models that consider watertightness and non-watertightness of vehicles during floods}

Oshikawa and Komatsu (2014) conducted experimental tests with 1:24 scale physical models of a Nissan March compact car and a 4WD Toyota Land Cruiser. From the results analysis the stability threshold was determined as the ratio between the drag force and the friction one. A value of this ratio greater than 1.0 indicates that the vehicle would be washed away by the water flow. 


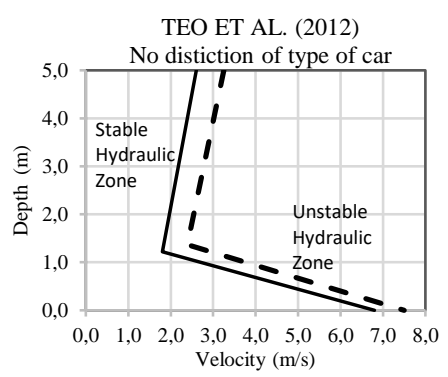

a.- Stability models that consider non-watertightness of vehicles
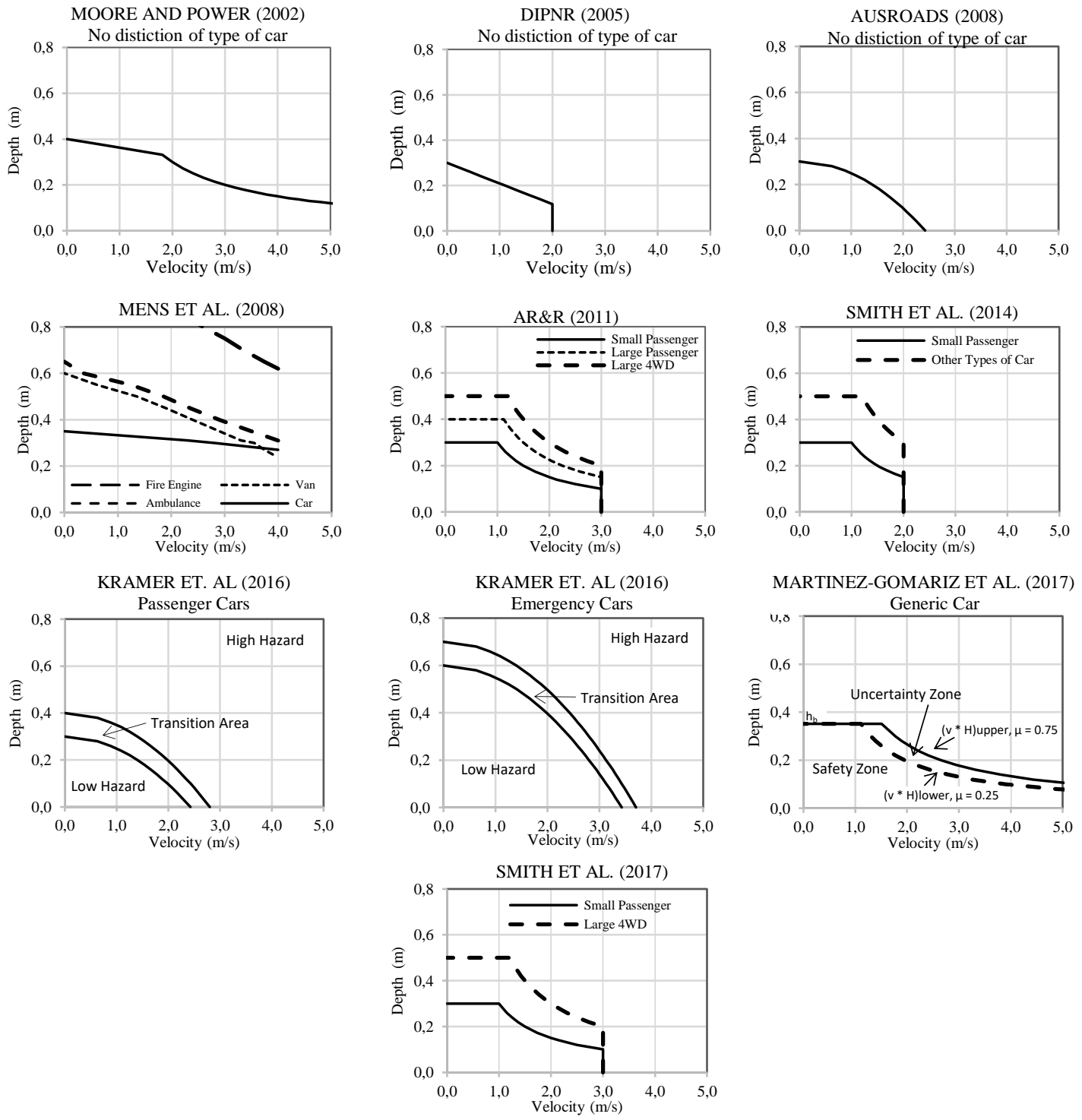

b.- Stability models that consider watertightness of vehicles
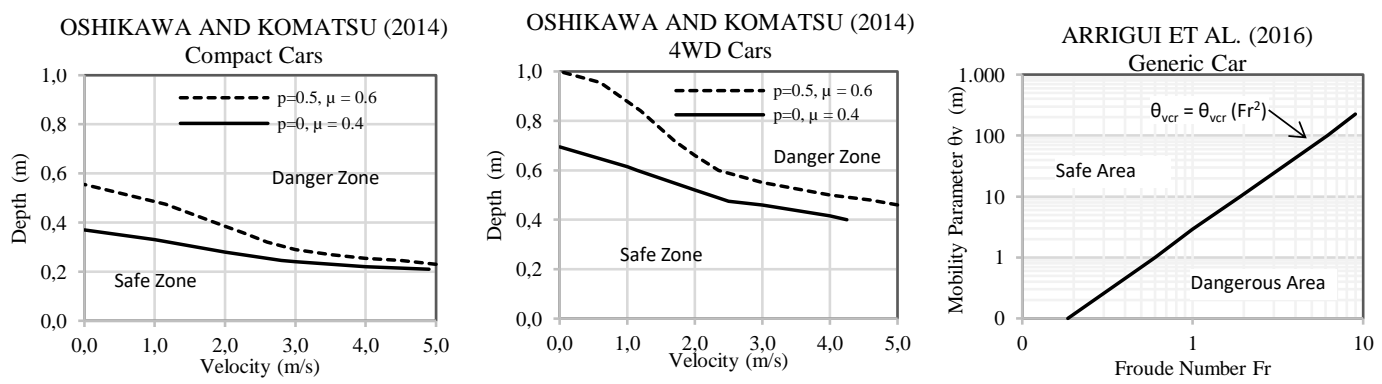

c.- Stability models that consider watertightness and non - watertightness of vehicles

Different scales have been used in panels $\mathrm{a}, \mathrm{b}$ and $\mathrm{c}$ for better visibility

Figure 1. Stability thresholds for vehicles in flood events 
Values of drag and lift coefficients were experimentally determined and used to calculate the corresponding forces exerted by the flow on the vehicle. The drag coefficients fluctuated between 0.8 and 5.1 for the compact car, and between 2.1 and 3.6 for the 4WD car. Lift coefficients varied between -0.28 and 0 for the compact vehicle and between -0.52 and -0.17 for the 4WD vehicle. In the cases where water can enter the vehicle, there will be a decrease in the flotation force due to the vehicle porosity, which was defined with a minimum value of 0.0 when the vehicle was well-closed and with a maximum value of 0.5 that corresponded to the cases where the dead weight of the vehicle and the buoyancy were balanced. Therefore, it is possible to define a safe zone below the obtained stability threshold with a friction coefficient of 0.4 and a porosity of 0.0 , and a danger zone above the result for a friction coefficient of 0.6 and a porosity of 0.5 (Oshikawa and Komatsu 2014) (Figure 1c).

Using a similar approach to that of Oshikawa and Komatsu (2014), Toda et al. (2013) performed laboratory tests using physical models at scale 1:10 of a sedan-style vehicle and 1:18 of a minivan.. These authors obtained friction coefficients equal to 0.26 for the sedan vehicle and 0.57 for the minivan with the car oriented in the flow direction and the handbrake on. With the car oriented transversely to the flow and with the handbrake off, the coefficients of friction were equal to 0.565 for the sedan vehicle and 0.65 for the minivan. Porosity values were established as $0.2,0.3$ and 0.5 . It was concluded that vehicles are likely to start moving with depths greater than $0.5 \mathrm{~m}$ and velocities greater than $2.0 \mathrm{~m} / \mathrm{s}$.

According to Arrighi et al. (2015), vehicle stability can be determined from the Froude number and a mobility parameter. This parameter is defined for water depths greater than the height of the chassis and considers the shape and the submerged relative weight of the vehicle. Arrighi et al. (2016a) improve the estimation of the mobility parameter when considering the incidence angle of the flow with the vehicle. The mobility parameter was calculated for experimental data reported by Xia et al. (2011), Shu et al. (2011) and Xia et al. (2014). The results were plotted against the corresponding Froude numbers, obtaining a stability threshold which determines a safe zone and a dangerous zone (Figure 1c). It should be noted that this model allows considering the entry of water into the vehicle during the flooding, because it allows modifying the density of the car, which is required to calculate the mobility parameter. Also, it is important to note that a certain degree of uncertainty is associated to the stability threshold, because Arrighi et al. used the data reported by Xia et al. (2011), which present the inaccuracies already discussed in subsection 2.1.

\section{COMPARISON OF VEHICLE STABILITY THRESHOLDS}

\subsection{Assuming vehicle watertightness}

Figure 2 compares the results obtained by applying the stability models that consider vehicle watertightness to three different types of cars according to the classification proposed by the AR\&R (Shand et al. 2011): large 4WD, large passengers and small passengers. Although the stability models of DIPNR (2005), Ausroads (2008) and Kramer et al. (2016) were proposed for any type of vehicle, they are only shown in the graph for small passenger vehicles, because, in reality, the vehicles they used fell within this category.

For the implementation of the stability models of Arrighi et al. (2016a) and MartínezGomariz et al. (2017), the following vehicles were used: (i) large 4WD cars: Mercedes G55 AMG and Audi Q7; (ii) large passenger cars: Mercedes GLA and Ford Focus; (iii) small passenger cars: Mini Cooper and Toyota Yaris. 

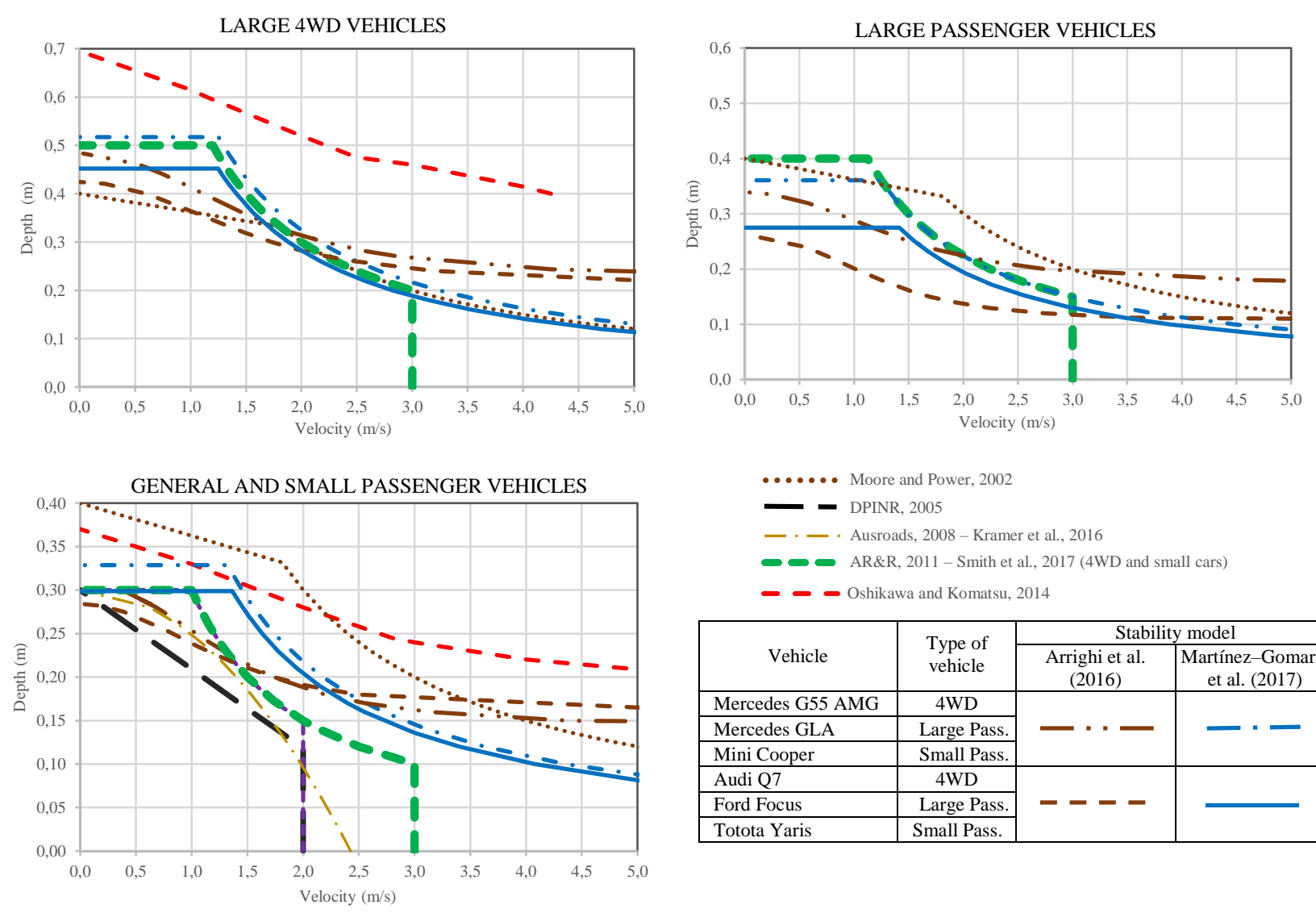

\begin{tabular}{|c|c|c|c|}
\hline \multirow[b]{2}{*}{ Vehicle } & \multirow{2}{*}{$\begin{array}{l}\text { Type of } \\
\text { vehicle }\end{array}$} & \multicolumn{2}{|c|}{ Stability model } \\
\hline & & $\begin{array}{l}\text { Arrighi et al. } \\
\text { (2016) }\end{array}$ & $\begin{array}{c}\text { Martínez-Gomariz } \\
\text { et al. (2017) }\end{array}$ \\
\hline Mercedes G55 AMG & 4WD & \multirow{3}{*}{$\because \because-$} & \multirow{3}{*}{$-\cdot$} \\
\hline Mercedes GLA & Large Pass. & & \\
\hline Mini Cooper & Small Pass. & & \\
\hline Audi Q7 & 4WD & \multirow{3}{*}{----} & \\
\hline Ford Focus & Large Pass. & & \\
\hline Totota Yaris & Small Pass. & & \\
\hline
\end{tabular}

Figure 2. Comparison of vehicle stability thresholds during floods proposed by stability models that consider car watertightness

The analysis of Figure 2 allows identifying how the studied stability models generate a wide range of stability thresholds, which are based on the decision criteria established in each case. For example, in the case of 4WD vehicles, with a flow velocity of $3.5 \mathrm{~m} / \mathrm{s}$, it can be observed that the stability models proposed by AR\&R (2011) and Smith et al. (2017) consider it is not safe to drive with any depth, while the models proposed by Moore and Power (2012) and Martínez-Gomariz et al. (2017) consider safe to drive with depths approximately equal to or less than 0.18 meters and Oshikawa and Komatsu (2014) establish this depth limit at approximately 0.45 meters. Variations of similar order of magnitude are observed for large and small passenger vehicles.

There are also differences in the shape of the safety thresholds provided by the different stability models, presenting different decreases in depth as flow velocity increases (Figure 2). Concerning high velocities, on the one hand, Arrighi et al. (2016a) do not establish a limit to flow velocity for the circulation of vehicles with depths lower than the chassis height. On the other hand, the stability models proposed by Martínez-Gomariz et al. (2017) and Moore and Power (2002) admit limits to flow velocity for low flow depths. The remaining models establish maximum velocities values between $3.0 \mathrm{~m} / \mathrm{s}$ and $5.0 \mathrm{~m} / \mathrm{s}$ to define the stability threshold.

From Figure 2, it can be underlined that the stability thresholds proposed by Moore and Power (2002) for velocities greater than $1.81 \mathrm{~m} / \mathrm{s}$, AR\&R (2011), Martínez-Gomariz et al. (2017) and Smith et al. (2017) are equal or quite similar to each other. The same similarity is 
observed among the stability models proposed by DIPNR (2005), Ausroads (2008) and Kramer et al. (2016), but for a different range of values.

\subsection{Assuming vehicle non-watertightness}

Figure 3 compares the results obtained when implementing the stability models that consider non-watertightness of the vehicles. Considering that the model proposed by Teo el al. (2012) establishes an instantaneous water entry into the vehicle, and in order to obtain comparable figures, this graph presents the values proposed by Oshikawa and Komatsu (2014) for an 4WD vehicle with porosity equal to 0.5 and the results obtained by Arrighi et al. (2016a) to a Mercedes G55 AMG car, which corresponds to a large 4WD vehicle. In the latter case, three scenarios were considered with different amounts of water entering the vehicle, increasing the weight of the car by 250,300 and $400 \%$. The increase in weight equal to $250 \%$ is approximately the same as the average increase considered in the experimental data of the 4WD car used by Teo et al. (2012).

The information shown in Figure 3 makes it possible to conclude that for low velocities the stability model proposed by Teo et al. (2012) establishes a stability threshold through the combination of flow velocities and depths that are considered unsafe or exceed several times the limit values proposed by the other stability models. In general, the stability thresholds proposed by Arrighi et al. (2016a) for a weight increase of 300\% and Oshikawa and Komatsu (2014) are quite similar to each other. For high velocities, Teo et al. (2012) consider that speeds higher than $6.8 \mathrm{~m} / \mathrm{s}$ are unsafe with any depth, while Arrighi et al. (2016a) do not set limits for low depths. The model proposed by Oshikawa and Komatsu (2014) only consider flow velocities lower than 5.0.

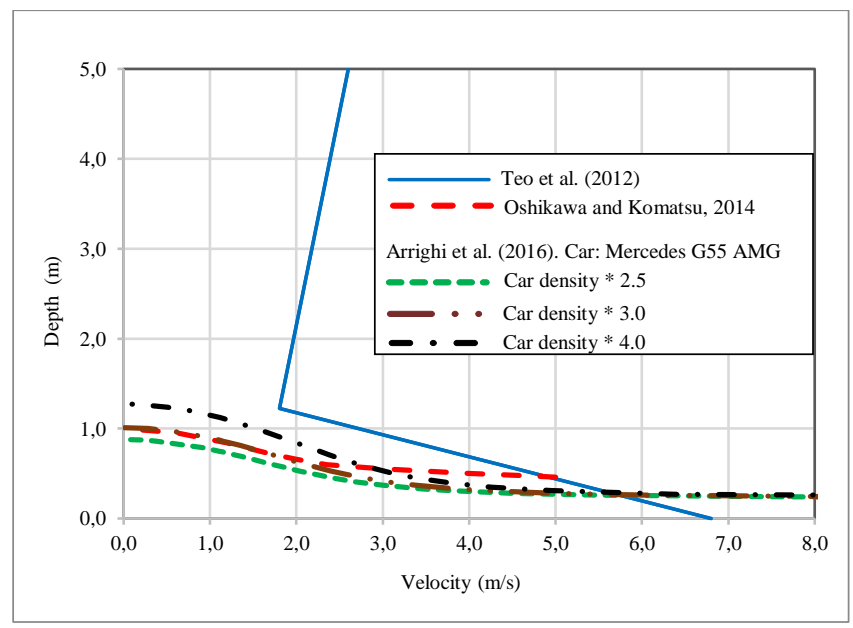

Note: The stability model of Arrighi et al. (2016a) was applied for a Mercedes G55 AMG car considering that the water volume getting inside the vehicle increases its density by a 250,300 and $400 \%$

Figure 3. Comparison of vehicle stability thresholds during floods proposed by models that consider large 4WD vehicles and car non-watertightness.

\section{COMPARISON OF VEHICLE STABILITY THRESHOLDS WITH EXPERIMENTAL DATA}

The goal of this Section is to compare experimentally obtained data of depth and flow velocity in which the vehicles studied under watertight conditions lost their stability with the 
ranges of values in which the thresholds of the previous stability models fluctuate for the three types of vehicles defined by the AR\&R in 2011 (Figure 4). In the determination of these ranges, the stability model of Moore and Power (2002) was not considered because they used experimental and analytical data for old cars, which had different characteristics from modern ones.

\subsection{Experimental data}

The results for experimental data are condensed in Figure 4 as dots. In general, it is observed in all cases that, as expected, the depths found experimentally show a tendency to decrease as flow velocity increases. However, this tendency seems to differ between data obtained in different laboratory tests, since the decrease in depths related to the increase in velocities is bigger in some measurements than in others. For example, in the data measured for large vehicles 4WD by Smith et al. in 2017, there is a much more pronounced decrease in the depths causing vehicle destabilization than the decrease observed in the data recorded by Shu et al. in 2011. In other cases, the depths descend rapidly until a certain velocity is reached and from that point onwards, this decrease is less pronounced (measurements made by MartínezGomariz et al. in 2016 for large passenger vehicles), which contrasts with other measurements in which the decrease in depths seems to have a more uniform tendency for the range of studied velocities (for example, measurements made by Shu et al in 2011 for large passenger vehicles).

Figure 4 also shows that experimental data have a relatively high sample dispersion. For example, Martínez-Gomariz et al. (2017) found that the Mercedes GLA reached conditions of instability with a flow velocity of $1.98 \mathrm{~m} / \mathrm{s}$ and a depth of $0.30 \mathrm{~m}$, while in the study carried out by Shu et al. (2011) it was found that the Ford Focus vehicle, which can be classified in the same category that the Mercedes GLA but it should be less stable, lost its stability at the same depth when the velocity reached a value of $4.0 \mathrm{~m} / \mathrm{s}$, that is, at twice the velocity found for the Mercedes GLA.

The sample dispersion found in the trends followed by the consulted experimental data could be due, in part, to the differences in the flow conditions of the flumes and the quality and scales of the physical models used in the tests carried out. The used flumes width varied between 0.6 and $1.2 \mathrm{~m}$, which implies that the sidewalls could have exerted an effect not considered in the results in the case of the narrower ones. This is due to the fact that, when cars were oriented in the normal direction to the flow, the walls could have been too close to the front and rear car ends, affecting their behaviour. Additionally, the flume bottoms were constituted by different materials among which are acrylic, bakelite, cement and plastic; due to this, the friction coefficient between the car wheels and the bottom of the flumes fluctuated in relatively wide range (Table 1), which generates variations in the stability thresholds.

On the other hand, scale models of different qualities have been used, such as: commercial plastic models, model produced by powder-based laser sintering, radio control models and diecast models. Not all of these models made an appropriate scaling of the geometric characteristics and the weight of the prototype vehicles, which could be one of the causes of the dispersion presented by the experimental results. In addition, the scales of the physical models used fluctuated between 1:9.8 and 1:24 (Table 1), which represents a relatively wide range and could generate important differences in the uncompensated scale effects that occur when working with this type of models. 
In all cases, the depths measured in the laboratory that generated vehicle stability loss were greater than the free height between the floor and the chassis. This could suggest that, as considered by the stability model proposed by Arrighi et al. (2016a), water depths lower than chassis height would not destabilize the vehicles within the studied velocity range, which corresponds approximately to the expected range in real situations.

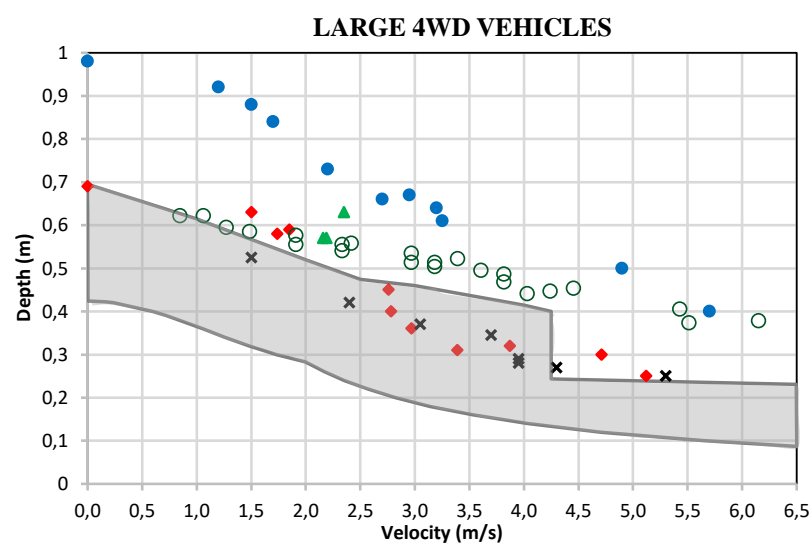

\begin{tabular}{|c|c|c|c|}
\hline \multicolumn{4}{|c|}{ Experimental Data } \\
\hline \multirow[b]{2}{*}{ Author } & \multicolumn{3}{|c|}{ Type of vehicle } \\
\hline & Large 4WD & Large passenger & $\begin{array}{c}\text { Small } \\
\text { passenger }\end{array}$ \\
\hline Shu et al. (2011) & O Volvo XC90 & $\begin{array}{l}\text { O Ford Transit } \\
\text { OFord Focus }\end{array}$ & \\
\hline Toda et al. (2013) & $\Delta$ Minivan & $\Delta$ Sedan & \\
\hline Xia et al. (2013) & * Audi Q7 & * Honda Accord & \\
\hline $\begin{array}{l}\text { Martínez- Gomariz et } \\
\text { al. (2017) }\end{array}$ & $\diamond$ Mercedes G55 AMG & Mercedes GLA & Mini Cooper \\
\hline Kramer et al. (2016) & & & * VW Golf III \\
\hline Smith et al. (2017) & - Nissan Pat. GRII & & Toyota Yaris \\
\hline
\end{tabular}

Range in which the proposed stability thresholds fluctuate
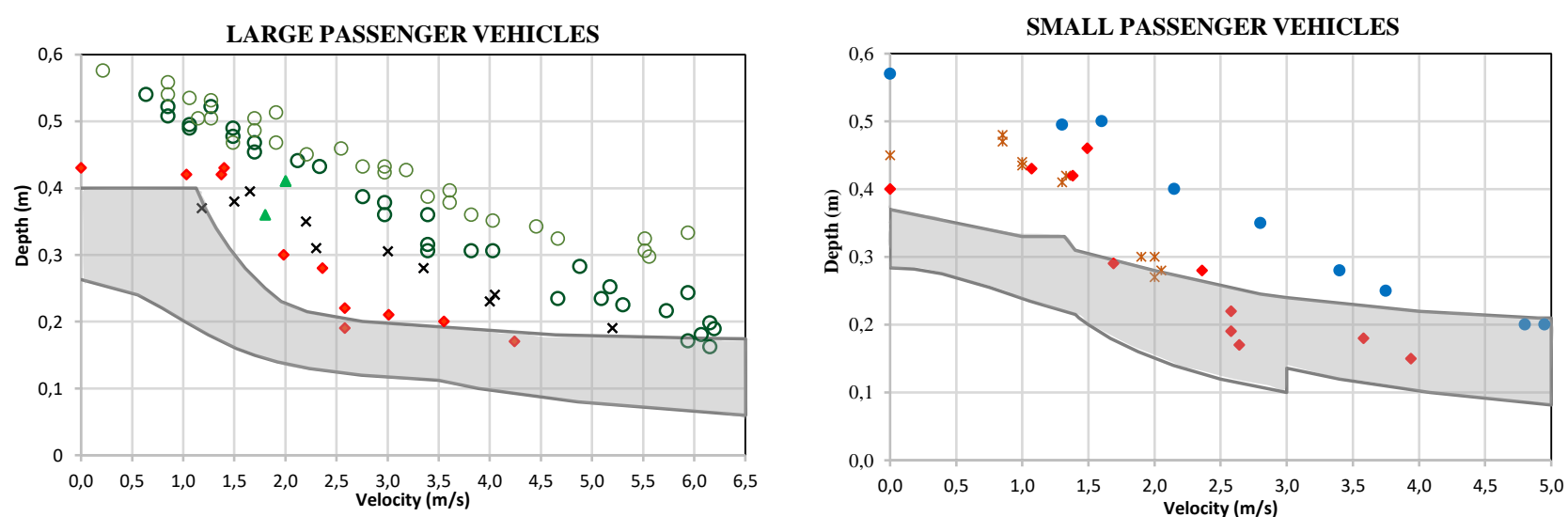

Figure 4. Comparison of proposed stability thresholds for vehicles under watertight conditions during floods with experimental data

\subsection{Comparison between experimental data and stability models}

For low flow velocities most of the stability models (grey area) seem to be too conservative, as the proposed thresholds are relatively far from the experimental measurements (Figure 4). For medium and high velocities, some of the experimental results start to fall within the range of the proposed stability thresholds, especially for small cars. This situation could suggest that some of the studied stability models, such as those of Oshikawa and Komatsu (2014) and Moore and Power (2012) for large and small passenger cars (as can be seen in previous Figure 3 ), propose combinations of flow velocity and depth that would not guarantee vehicle stability during flood events. For high velocities some of the stability models seem too conservative. For example, for reasons of passenger safety when leaving vehicles, the model of the AR\&R (2011) considers it unsafe to circulate at any depth when the velocities are higher than $3.0 \mathrm{~m} / \mathrm{s}$ (Not represented in figure 4) and the model of Martínez-Gomariz et al. (2017) admits stable depths that can be considered too low when compared with the experimental ones.

On the other hand, the stability models proposed by DIPNR (2005), Austroads (2008) and Kramer et al. (2016) (Figure 1b) seem to be somewhat conservative for large passenger cars and very conservative for $4 \mathrm{WD}$ vehicles, since the data found experimentally (Figure 4) are quite different from the stability thresholds proposed by these models. In the case of the 
stability model proposed by Kramer et al. (2016), this could be due to the fact that the stability threshold was determined from the analysis of experimental results obtained for one single small passenger vehicle.

\section{CONCLUSIONS}

In recent years several authors have proposed different models to establish vehicle stability thresholds during a flood event, most of them have considered that vehicles are watertight, some have considered that water can enter the vehicles exposed to flooding and others allow considering both watertightness and non-watertightness conditions. Also, the criterion to determine the stability threshold varies among the studied stability models. These differences in the way of approaching vehicle watertightness, the decision criterion adopted to determine the stability of the cars and, more importantly, different driving factors, lead to quite a wide range of stability thresholds as obtained by the various models.

All the stability models have made simplifications in the experimental part or in the theoretical deduction of the stability thresholds that can influence the final result. Some of the main simplifications are the following:

1) Only cars at rest have been considered;

2) Most of the experiments have been carried out on a horizontal surface;

3) The friction coefficient between the tyres and the road has not been studied in depth, especially when considering it can vary during flood events;

4) The actual weight distribution of the vehicles (with greater weight in the front part due to the location of the engine) has not been considered in several of the developed studies;

5) The tests have been conducted using a controlled flow. This is not representative of reallife flow conditions, which could be, for example, variable or pulsating;

6) Measurements have been made in laboratory flumes, whose characteristics can vary significantly from the conditions of the actual roads;

7) Most of the experimental studies have been carried out with scale physical models, implying that some forces acting on the vehicles may not have been well represented due to scale effects;

8) With the exception of the study developed by Martínez-Gomariz et al. (2017), experimental tests have made measurements on very few cars, which were not always the most vulnerable ones to flooding for each vehicle category.

With regard to the theoretical approach of the studied stability models, it should be noted that the one developed by Arrighi et al. (2016a) combines several aspects that can make it one of the most robust. Among these aspects, it is worth highlighting that the equations proposed are based on a solid theoretical base and that include the use of Froude number, which is a very important and widely used parameter in many formulations. In addition, this methodology allows to consider simultaneously watertightness and non-watertightness conditions of the vehicles and enables the calculation of a stability threshold for any vehicle considering key factors as ground clearance and car density.

The comparison of the studied stability models with the experimental data suggests that most stability models seem to be conservative for low flow velocities and that the models proposed by the DIPNR (2005), Ausroads (2008), Kramer et al. (2016), Martínez-Gomariz et al. (2017) for high velocities and the AR\&R (2011) and Smith (2017) for speeds over $3.0 \mathrm{~m} / \mathrm{s}$ seem to be excessively conservative. Additionally, it could be observed that the stability models 
proposed by Moore and Power (2002) for small passenger cars and by Oshikawa and Komatsu (2014) establish limits of stability threshold higher than the combinations of velocity and depth for which several of the studied experimental cars lost their stability. Because of this, these models could be unsafe for certain types of car.

The stability model proposed by Arrighi et al. (2016a) seems to provide an acceptable fit for the experimental data for medium and high velocities while the model proposed by MartínezGomariz seems to have a similar goodness of fit for the measured data in the case of medium velocities. It should be highlighted that these models are the only ones that allow calculating a stability threshold for any vehicle.

To date, numerous experiments have been conducted with very productive results, which have been used to establish different vehicle stability models. However, the experimental data and the stability models present excessive sample dispersion. This is why, we consider it necessary to conduct new research that focuses on:

1) Overcoming the simplifications that have been made so far, as some of them are too restrictive so the tests performed could have produced results that are not sufficiently representative of the stability of vehicles against floods.

2) Accompanying the laboratory experiments with the development of mathematical modelling of the vehicle-flow interaction, similar to that done by Arrighi et al. in 2015, since this can contribute to a better understanding of the hydrodynamic phenomena that cause vehicle stability loss.

3) Trying to standardize the decision criterion that must be adopted to define the stability thresholds. The aspects that have a greater impact on the stability of the vehicles should be better identified and laboratory tests and theoretical analyses should be carried out with greater emphasis on these aspects.

4) Performing more experiments on a representative number of vehicles of various characteristics, including the most vulnerable ones in each car category. Also, some kind of safety factor could be considered.

5) Establishing the friction coefficients between the tyres and the surface by conducting tests with real-scale vehicles and variable water depths. These tests should be carried out considering different surface materials and tyres in different wear conditions.

6) Finally, and probably the most difficult to accomplish recommendation due to the environmental legislation: carrying out experiments at 1:1 scale in which the vehicles lose their stability. Since making these measurements in laboratory flumes is very complex (because very large flows and huge flumes would be required), at least these tests could be performed in tanks with water at rest to study the flotation and leaking of water inside the vehicles, increasing the number of experiments done by Kramer et al. in 2016 and Smith et al. in 2017. In order to include the full-scale flow velocity, experiments could be carried out downstream of dams, as also suggested by Xia et al. in 2011.

The conduction of new studies and laboratory tests that include these aspects, at least to some extent, would allow reducing the present uncertainty and would give the possibility of formulating more generalized and robust vehicle stability models.

\section{ACKNOWLEDGEMENTS}


This work was supported by COLCIENCIAS call 728 - 2015 (Colombia) and by the Spanish Ministry of Science and Innovation through the research project TETISCHANGE (ref. RTI2018-093717-B-I00).

\section{REFERENCES}

Arrighi C., Alcérreca - Huerta J., Oumeraci H., Castelli F. (2015). Drag and lift contribution to the incipient motion of partly submerged flooded vehicles. JFluids Struc, 57, 170 184. DOI: $10.1016 /$ j.jfluidstructs.2015. 06.010

Arrighi C., Castelli F., Oumeraci H., (2016a). Effects of flow orientation on the onset of motion of flooded vehicles. Proceedings of the 4th IAHR Europe Congress. Liege, DOI: 10.1201/b21902-140.

Arrighi C., Huybrechts N., Ouahsine A., Chaseé P., Oumeraci H., Castelli F. (2016b). Vehicles instability criteria for flood risk assessment of a street network. Proceedings of the IAHS, 373, pp. 143-146, DOI:10.5194/piahs-373-143-2016.

Austroads (2008). Guide to road design, Part 5: Drainage design. AusRoads Inc., Australia.

Bonham A. J., Hattersley R. T. (1967). Low level causeways. WRL Report No. 100. University of New South Wales. Sydney, Australia.

Cox R. J., Shand T. D. and Blacka, M. J. (2010). Appropriate safety criteria for people in floods. Australian Rainfall and Runoff. WRL Research Report 240. Report for Institution of Engineers Australia.

Department of Infrastructure, Planning and Natural Resources DIPNR (New South Wales Government) (2005). Floodplain development manual: The management of flood liable land. Sydney, Australia.

Drobot, S.D., Benight, C., Gruntfestd, E.C. (2007). Risk factors for driving into flooded roads. Environmental Hazards, 7 (3), 227-334. DOI:10.1016/j.envhaz.2007.07.003

Fitzgerald, G., Du, W., Jamal, A., Clark, M., and Hou, X. (2010). Flood fatalities in contemporary Australia (1997-2008), Emerg. Med. Australas, 22, 180-186. DOI: $10.1111 / \mathrm{j} .1742-6723.2010 .01284 . \mathrm{x}$

Gordon A. D., Stone P. B. (1973) Car stability on road causeways. WRL Technical Report No. 73/12. University of New South Wales. Sydney.

Jonkman, N., Kelman, I. (2005). An analysis of the causes and circumstances of flood disaster deaths. Disasters, 29(1), 75 -97.

Kalantari Z., Nickman A., Lyon S., Olofsson B., Folkeson L. (2014). A method for mapping flood hazard along roads. Environ Manage, 133, 69-77. DOI:10.1016/j.jenvman.2013.11.032.x. 
Kellar. M. \& Schmidlin, T. W. (2012). Vehicle-related flood deaths in the United States, 1995-2005. Journal of Flood Risk Management, 5, 153- 163. DOI: 10.1111/j.1753318X.2012.01136.X.

Keller R. J., Mitsch B. (1993). Safety aspects of the design of roadways as floodways. Research Report No. 69, Urban Water Research Association of Australia.

Kramer M., Terheiden K., Wieprecht S. (2016). Safety criteria for trafficability of inundated roads in urban floodings. Int $J$ Disaster Risk Reduct, 17, 77 - 84. DOI:10.1016/j.ijdrr.2016.04.003.

Martínez-Gomariz E., Gómez M., Russo B., Djordjević S. (2016). Stability criteria for flooded vehicles: a state-of-the-art review. Journal of Flood Risk Management. DOI:10.1111/jfr3.12262

Martínez-Gomariz E., Gómez M., Russo B., Djordjević S. (2017). A new experiments-based methodology to define the stability threshold for any vehicle exposed to flooding. Urban Water J, 14(9), 930-939. DOI: 10.1080/1573062X.2017.1301501.

Mens M. J., Erlich M., Gaume E., Lumbroso D., Moreda Y., Van der Vat M., Versini P. A. (2008). Frameworks for flood event management. Report Number T19-07-03. WL Delft Hydraulics. Delft.

Moore K. A., Power R. K. (2002). Safe buffer distances for offstream earth dams. Australasian Journal of Water Resources, 6:1, 1-15. DOI: 10.1080/13241583.2002.11465206.

Oshikawa H. \& Komatsu T. (2014). Study on the risk evaluation for a vehicular traffic in a flood situation. Proceedings of the 19th IAHR-APD Congress, Hanoi.

Pregnolato M., Ford A., Wilkinson S.M., Dawson R.J. (2017). The impact of flooding on road transport: A depth - disruption function. Transportation Research Part D, 55, 67-81. DOI: 10.1016/j.trd.2017.06.020.

Shand T., Cox R., Blacka M., Smith G. (2011). Australian Rainfall and Runoff (AR\&R). Appropriate safety criteria for vehicles. Australian rainfall and runoff, revision project 10 : Report Number: P10/S2/020. Sidney, Australia.

Shu, C., Xia, J., Falconer, R.A. and Lin, B. (2011). Incipient velocity for partially submerged vehicles in floodwaters. J Hydraul Res, 49 (6), 709-717. DOI:10.1080/00221686. 2011.616318

Smith G. P., Davey E. K., Cox R. J. (2014). Flood hazard. WRL Technical Report 2014/07. University of New South Wales. Sydney, Australia.

Smith G. P., Modra B. D., Tucker T. A., Cox R. J. (2017). Vehicle stability testing for flood flows. WRL Technical Report 2017/07. University of New South Wales. Sydney, Australia.

Suárez, P., Anderson, W., Mahal, V., Lakshmanan, T. (2005). Impacts of flooding and climate change on urban transportation: a system wide performance assessment of the Boston Metro Area. Transportation Research Part D, 10, 231-244. DOI:10.1016/ j.trd.2005.04.07. 
Teo Y., Falconer R., Lin B., Xia J. (2012a). Investigations of hazard risks relating to vehicles moving in flood. Water Resources Manag, 1, 52 -66.

Teo Y., Xia J., Falconer R., Lin B. (2012b). Experimental studies on the interaction between vehicles and floodplain flows. Intl. J River Basin Management, 10 (2), 149-160. DOI: 10.1080/15715124.2012.674040

Toda K., Ishigaki T., Ozaki T. (2013). Experiment study on floating car in flooding. International Conference on Flood Resilience Experiences in Asia and Europe. Exeter.

Versini, P. A., Gaume, E., Andrieu, H. (2010a) Assessment of the susceptibility of roads to flooding based on geographical information-test in a flash flood prone area (the Gard region, France). Nat Hazard Earth Sys, 10, 793-803.

Versini P.A., Gaume E., Andrieu H. (2010b). Application of a distributed hydrological model to the design of a road inundation warning system for flash flood prone areas. Nat Hazard Earth Sys, 10, 805 - 817.

Xia, J., Teo, F., Lin, B., Falconer, R. (2011). Formula of incipient velocity for flooded vehicles. Nat Hazard, 58 (1), 1-14. DOI: 10.1007/s11069-010-9639-x.

Xia J., Falconer R. A., Xiao X., Wang Y. (2014). Criterion of vehicle stability in floodwaters based on theoretical and experimental studies. Nat Hazards, 70 (2): 1619-1630. DOI: $10.1007 / \mathrm{s} 11069-013-0889-2$.

\section{WORD COUNT: 5960}

\section{FIGURE LEGENDS}

Figure 1 Stability thresholds for vehicles in floods

Figure 2. Comparison of vehicle stability thresholds during floods proposed by stability models that consider car watertightness

Figure 3. Comparison of vehicle stability thresholds during floods proposed by models that consider large 4WD vehicles and car non-watertightness

Figure 4. Comparison of proposed stability thresholds for vehicles under watertight conditions during floods with experimental data 
Table 1 Studied models for the determination of vehicle stability

\begin{tabular}{|c|c|c|c|c|c|c|c|c|c|c|c|}
\hline $\begin{array}{l}\text { Water- } \\
\text { tightness of } \\
\text { vehicles }\end{array}$ & Author & Year & Used data & \begin{tabular}{|c|} 
Scale \\
physical \\
models
\end{tabular} & $\begin{array}{l}\text { Velo- } \\
\text { city } \\
(\mathbf{m} / \mathbf{s})\end{array}$ & $\begin{array}{l}\text { Depth } \\
\text { (m) }\end{array}$ & $\begin{array}{l}\text { Froude } \\
\text { Number }\end{array}$ & $\begin{array}{c}\text { Friction } \\
\text { Coeffic. } \\
\mu\end{array}$ & $\begin{array}{l}\text { Drag. } \\
\text { Coeffic. }\end{array}$ & $\begin{array}{c}\text { Angle of } \\
\text { flow } \\
\beta^{(1)}\end{array}$ & Stability Equation ${ }^{(2)}$ \\
\hline $\begin{array}{l}\text { Non-water- } \\
\text { tightness }\end{array}$ & Teo et al. & 2012 & $\begin{array}{l}\text { Measurements on Mini Cooper, BMW M5, Mitsubishi } \\
\text { Pajero }\end{array}$ & $\begin{array}{l}1: 43 \\
1: 18\end{array}$ & $\begin{array}{c}2.37- \\
7.94\end{array}$ & $\begin{array}{c}0.65- \\
4.82\end{array}$ & $\begin{array}{c}0.50- \\
3.16\end{array}$ & - & - & $90^{\circ}-180^{\circ}$ & Linear relationship between $\mathrm{H}$ and $\mathrm{V}$ \\
\hline \multirow{7}{*}{$\begin{array}{l}\text { Water- } \\
\text { tightness }\end{array}$} & $\begin{array}{l}\text { Moore and } \\
\text { Power }\end{array}$ & 2002 & $\begin{array}{l}\text { Measurements reported by Bonham and Hettersley (1967), } \\
\text { Gordon and Stone (1973) and Keller and Mitsch (1993) }\end{array}$ & $\begin{array}{l}1: 16 \\
1: 25\end{array}$ & $\begin{array}{c}0.48- \\
3.69\end{array}$ & $\begin{array}{c}0.025- \\
0.57\end{array}$ & - & $0.3-1.0$ & - & $0^{\circ}, 90^{\circ}$ & $\begin{array}{c}\mathrm{H} \leq(0,4-0,0376 \mathrm{~V}) \text { for } \mathrm{V} \leq 1,81 \\
\mathrm{H}^{*} \mathrm{~V} \leq 0,6 \text { for } \mathrm{V}>1,81\end{array}$ \\
\hline & \begin{tabular}{|l|l|l|l} 
DIPNR \\
\end{tabular} & 2005 & Analysis of laboratory tests not specified & - & - & - & - & - & - & - & $\mathrm{V}<-11 * \mathrm{H}+3,3 \mathrm{~V} \leq 2,0$ \\
\hline & AR\&R & 2011 & $\begin{array}{l}\text { Measurements reported by Bonham and Hettersley (1967), } \\
\text { Gordon and Stone (1973) and Keller and Mitsch }(1993)^{(3)}\end{array}$ & $\begin{array}{l}1: 16 \\
1: 25\end{array}$ & $\begin{array}{l}0.48- \\
3.69\end{array}$ & $\begin{array}{c}0.025- \\
0.57\end{array}$ & - & $\begin{array}{c}0.3- \\
1.0\end{array}$ & - & $0^{\circ}, 90^{\circ}$ & $\begin{array}{lll}\text { Small cars: } H^{*} \mathrm{~V} \leq 0,3 & \mathrm{H} \leq 0,3 & \mathrm{~V} \leq 3,0 \\
\text { Large cars: } \mathrm{H}^{*} \mathrm{~V} \leq 0,45 & \mathrm{H} \leq 0,4 & \mathrm{~V} \leq 3,0 \\
\text { 4WD cars: } \mathrm{H}^{*} \mathrm{~V} \leq 0,6 & \mathrm{H} \leq 0,5 & \mathrm{~V} \leq 3,0\end{array}$ \\
\hline & Smith et al. & 2014 & $\begin{array}{l}\text { Analysis of stability thresholds reported by AR\&R in } 2011 \\
\text { for vehicles and in } 2010 \text { for people }\end{array}$ & - & - & - & - & - & - & - & $\begin{array}{lll}\text { Small cars: } H^{*} \mathrm{~V} \leq 0,3 & \mathrm{H} \leq 0,3 & \mathrm{~V} \leq 2,0 \\
\text { Others cars: } \mathrm{H}^{*} \mathrm{~V} \leq 0,6 & \mathrm{H} \leq 0,5 & \mathrm{~V} \leq 2,0\end{array}$ \\
\hline & Kramer et al. & 2016 & Measurements made on a VW Golf III and a LF 10/6 & $\begin{array}{c}1: 1 \\
1: 9.8 \\
1: 13.1\end{array}$ & $\begin{array}{l}0- \\
3.2\end{array}$ & $\begin{array}{c}0- \\
0.73\end{array}$ & $0-1.5$ & 0.3 & - & $\begin{array}{l}0^{\circ}, 45^{\circ} \\
\quad 90^{\circ}\end{array}$ & $\begin{array}{l}\text { Passengers cars: } \mathrm{H}+\mathrm{V}^{2} / 2 \mathrm{~g} \leq 0,3 \\
\text { Emergency cars: } \mathrm{H}+\mathrm{V}^{2} / 2 \mathrm{~g} \leq 0,6\end{array}$ \\
\hline & Smith et al. & 2017 & $\begin{array}{l}\text { Measurements on a Toyota Yaris } 2005 \text { and } 2006 \text { and a } \\
\text { Nissan Patrol } 1998\end{array}$ & $\begin{array}{l}1: 1 \\
1: 18 \\
\end{array}$ & $\begin{array}{c}0.80- \\
6.56\end{array}$ & $\begin{array}{c}0- \\
0.828\end{array}$ & $\begin{array}{c}0.30- \\
6.63 \\
\end{array}$ & $\begin{array}{c}0.75- \\
0.78\end{array}$ & $\begin{array}{c}0.98- \\
2.02 \\
\end{array}$ & $90^{\circ}$ & $\begin{array}{lll}\text { Small cars: } H^{*} V \leq 0,3 & H \leq 0,3 & \mathrm{~V} \leq 3,0 \\
\text { 4WD cars: } H^{*} \mathrm{~V} \leq 0,6 & \mathrm{H} \leq 0,5 & \mathrm{~V} \leq 3,0 \\
\end{array}$ \\
\hline & $\begin{array}{l}\text { Martínez- } \\
\text { Gomariz et } \\
\text { al. }\end{array}$ & 2017 & $\begin{array}{l}\text { Measurements made on BMW 650, Mini Cooper, BMW } \\
\text { i3, Mercedes GLA, Mercedes Class C, Range Rover } \\
\text { Evoque, Porsche Cayenne Turbo, Bentley Continental } \\
\text { GT, Volkswagen Touareg, BMW X6, Audi Q7, Mercedes } \\
\text { G55 AMG }\end{array}$ & $\begin{array}{l}1: 14 \\
1: 18 \\
1: 24\end{array}$ & $\begin{array}{r}0.89 \\
5.12\end{array}$ & $\begin{array}{c}0.16- \\
0.63\end{array}$ & - & $\begin{array}{c}0.25- \\
0.75\end{array}$ & - & $\begin{array}{l}\text { All } \\
\text { orientations } \\
\text { were } \\
\text { considered }\end{array}$ & $\begin{array}{c}H * V=0,0158 * \frac{G_{c} * M_{c}}{P A} * \mu+0,32 \\
H<h_{b}=\frac{M_{c}}{\rho * L * l}+G_{c}\end{array}$ \\
\hline \multirow{3}{*}{$\begin{array}{l}\text { Water- } \\
\text { tightness/ } \\
\text { Non- Water- } \\
\text { tightness }\end{array}$} & Toda et al. & 2013 & $\begin{array}{l}\text { Measurements made with a sedan-type vehicle and a } \\
\text { minivan (ambulance) }\end{array}$ & $\begin{array}{l}1: 10 \\
1: 18\end{array}$ & $\begin{array}{c}1.05- \\
2.00\end{array}$ & $\begin{array}{c}0.30- \\
1.21\end{array}$ & - & $\begin{array}{c}0.26- \\
0.65\end{array}$ & $\begin{array}{c}0.40- \\
3.50\end{array}$ & $\begin{array}{l}0^{\circ}, 45^{\circ} \\
90^{\circ}\end{array}$ & $\mathrm{V} \leq 2,0$ and $\mathrm{H} \leq 0,5$ \\
\hline & \begin{tabular}{|l|} 
Oshikawa \\
and Komatsu
\end{tabular} & 2014 & $\begin{array}{l}\text { Measurements made on a Nissan March and a Toyota } \\
\text { Land Cruiser }\end{array}$ & $1: 24$ & $\begin{array}{c}1.47- \\
7.35\end{array}$ & $\begin{array}{c}0.24- \\
1.08\end{array}$ & $\begin{array}{c}0.90- \\
2.77\end{array}$ & $\begin{array}{c}0.40- \\
0.60\end{array}$ & $\begin{array}{c}0.75- \\
5.1\end{array}$ & $90^{\circ}$ & Non-linear relationship between $\mathrm{H}$ and $\mathrm{V}$ \\
\hline & Arrighi et al. & 2016 & $\begin{array}{l}\text { Measurements reported by Shu et al. (2011), Xia et al. } \\
\text { (2011) and Xia et al. (2014) }\end{array}$ & $\begin{array}{l}1: 14 \\
1: 18 \\
1: 43\end{array}$ & $\begin{array}{c}0.18- \\
7.94\end{array}$ & $\begin{array}{r}0.11- \\
4.82\end{array}$ & $\begin{array}{l}0.50- \\
3.16^{(4)}\end{array}$ & $\begin{array}{c}0.25- \\
0.75\end{array}$ & - & $\begin{array}{c}0^{\circ}, 90^{\circ} \\
180^{\circ}\end{array}$ & 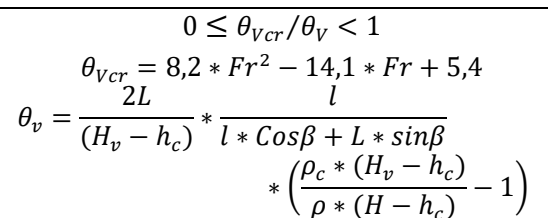 \\
\hline
\end{tabular}

(1) $\beta=$ angle of incidence of the flow with respect to the vehicle: $0^{\circ}=$ Vehicle oriented in the direction of flow and with the front facing the flow; $90^{\circ}=$ Vehicle oriented in the direction perpendicular to the flow; $180^{\circ}=$ Vehicle oriented in the direction of flow and with the rear facing the flow
(2) $\mathrm{H}=$ Depth (m)
$\mathrm{V}=$ Flow velocity $(\mathrm{m} / \mathrm{s})$
$\mathrm{Gc}=$ Ground clearance $(\mathrm{m})$
$\mathrm{Mc}=$ Weight $(\mathrm{kg})$
$\mathrm{PA}=$ Plan Area $\left(\mathrm{m}^{2}\right)$
$\mu=$ Friction Coefficient
$\rho=$ Water density $\left(\mathrm{kg} / \mathrm{m}^{3}\right)$
$\mathrm{L}$ = Length of the vehicle $(\mathrm{m})$
1 = Width of the vehicle $(\mathrm{m})$
$\theta_{\text {vcr }}=$ Critical threshold
(3) These measurements were
$\mathrm{Hv}=$ Height of the vehicle (m)
hc $=$ Height of the planform (m)
$\rho c=$ Car density $\left(\mathrm{kg} / \mathrm{m}^{3}\right)$

(4) Only reported by Xia (2011) 
